\title{
Optical Observations of Lensed Quasars: Multiwavelength Correlations and Interband Time Delays
}

\author{
Ekaterina Koptelova $^{1,2, *}$ and Victor Oknyanskij ${ }^{2}$ \\ ${ }^{I}$ Graduate Institute of Astronomy of National Central University, Jhongda Rd., Jhongli City, Taoyuan County 320, \\ Taiwan \\ ${ }^{2}$ Sternberg Astronomical Institute of Moscow University, Universitetski pr. 13, 119992 Moscow, Russia
}

\begin{abstract}
The time delays between brightness variations in different optical bands have been measured for a large number of Seyfert galaxies and low-redshift quasars. These time delays represent the travel time of light between different emission regions of the source and can be used to test variability scenarios. Based on multiwavelength observations of the lensed quasars: Q2237+0305, SBS1520+530, HE2149-2745, HE1104-1805 and UM673, we explore a variability mechanism in the redshift range $1.4 \leq z \leq 2.7$. From cross-correlation analysis of the light curves of the quasars we find that the brightness variations at longer wavelengths may follow the brightness variations at shorter wavelengths by a few days. An independent analysis of the multiband light curves of nonlensed quasars from the same redshift interval confirms the evidence of a time delay. A comparison of the observed time delays with the delays expected from a model of an accretion disc irradiated by the central variable source shows that reprocessing might be the primary mechanism responsible for the observed variability on the considered timescales.
\end{abstract}

Keywords: Gravitational lensing - quasars, accretion discs, observations.

\section{INTRODUCTION}

Active galactic nuclei (AGN) are known to be variable throughout the electromagnetic spectrum. The brightness variations at the UV/optical wavelengths are often wellcorrelated, sometimes with measurable time delays. This was clearly shown in the first multiwavelength monitoring observations of the Seyfert galaxies NGC4151 and NGC5548 [1, 2]. The correlated variations in different bands suggest a common mechanism of variability. One possible explanation is that variations in the UV/optical continuum are driven by reprocessing of the X-ray radiation from the central part of the accretion disc [3]. In this case, the variations at optical wavelengths would be expected to lag behind the X-ray variations. The most recent simultaneous $\mathrm{X}$-ray/optical observations of the Seyfert galaxies and lowredshift quasars confirm that reprocessing of the X-ray radiation plays an important role in AGN variability (see [47]). At the same time, the interband relations might be very complex and sometimes can not be explained by the reprocessing model alone. Other mechanisms, such as accretion disc instabilities which can drive the X-ray variations via Comptonisation, the presence of a weak blazar component (see $[8,9])$ or disc fluctuations drifting inward [4] are also considered to explain the multiwavelength correlations. Thus, the observed correlations between different bands and interband time delays can give important constraints on the origin of AGN variability. If reprocessing is the primary mechanism responsible for the variability,

*Address correspondence to this author at the Graduate Institute of Astronomy of National Central University, Jhongda Rd., Jhongli City, Taoyuan County 320, Taiwan; Tel: +886-3-4227151; Fax: +886-3-4262304; E-mail: koptelova@astro.ncu.edu.tw then one would expect that variations at longer wavelengths lag behind the variations at shorter wavelengths.

The first evidence of such a time delay was found by Collier et al. for NGC7469 [10]. Later, similar time delays between brightness variations in different optical bands were measured for NGC4151 [11]. Sergeev et al. detected interband delays between variations in the optical bands for 14 nearby Seyfert galaxies [12]. The correlations between variations in the optical bands were studied in a sample of quasars $(0.2<z<2.8)$ from the MACHO database (see [13]) and in a sample of low-redshift quasars $(z<1)$, optically monitored at the Wise Observatory (see $[14,15]$ ). Although the wavelength intervals between optical bands were small, some of the quasars showed possible time delays, with variations at bluer wavelengths leading the variations at redder wavelengths. The multiwavelength observations of lensed quasars can help us to explore mechanisms of quasar variability at higher redshifts $(z>1)$. The brightness changes of images of the lensed quasars occur as a result of intrinsic quasar variability, as well as due to microlensing by the stars in the lensing galaxy. In the first case, the light curves of the quasar images should be similar to each other, apart from a magnitude offset and a lensing time delay. The brightness changes due to microlensing occur independently in each quasar image. Given that the lensing time delay is known, it is possible to distinguish between these two causes of brightness variations. Refsdal showed that the time delay between quasar's intrinsic variations in the images of the lensed system can give us an estimate of the Hubble constant [16]. Hence, the main goal of the ongoing monitoring observations of lensed quasars in the optical/NIR bands is to measure lensing time delays. Much less attention is paid to the analysis of the interband 
correlations and investigation of the origin of the quasar variability. The first interband time delay in the lensed quasar was detected between brightness variations in the $\mathrm{g}$ and r-band light curves of Q0957+561 [17]. It was found to be consistent with the delay expected from reprocessing in a standard accretion disc irradiated by an X-ray source. The new $g$ and r-band observations confirm the existence of this delay [18]. The evidence of a time delay between brightness variations in the $\mathrm{V}$ and $\mathrm{R}$ bands has also been found in the lensed quasar Q2237+0305 [19].

In the present study, we analyse observational data of a sample of lensed quasars monitored in two or more optical bands with the aim of measuring possible interband time delays. To make our analysis more complete, we also explore the interband correlations in nonlensed quasars from the MACHO database, covering the same redshift range as the lensed quasars. Finally, we compare observed time delays with the delays expected from reprocessing in the standard accretion disc.

\section{MODEL PREDICTIONS: IRRADIATED ACCRE- TION DISC}

In order to estimate expected interband time delays, we consider a model which accounts both for intrinsic emission from the disc and reprocessing of the X-ray emission, namely the standard accretion disc of Shakura and Sunyaev [20] irradiated by a central variable source of X-ray radiation. The source irradiates the outer region of the accretion disc producing UV/optical continuum variability. As a result of irradiation the structure of the disc is modified, so that the resulting temperature profile is determined both by the viscous dissipation and irradiation

$T(R)=\left[\frac{3 G M \dot{M}}{8 \pi R^{3} \sigma}+\frac{(1-A)}{\sigma} F(R)\right]^{1 / 4}$,

where the first and second terms describe the temperature due to viscous $T_{\text {vis }}$ and irradiative heating $T_{\mathrm{i} r r}$, respectively

[21]. $M$ is the black hole mass; $\dot{M}$ is the mass accretion rate at radius $R ; A$ is the albedo of the disc surface; $F(R)$ is the incident flux of the X-ray source on to the disc at radius $R$. The relation between flux $F$ and radius depends on the model used to describe the source of irradiation. The models discussed include those that lead to a similar dependance of $T_{\text {vis }}$ and $T_{\mathrm{i} r r}$ on radius. For examples, Berkley et al. [22] considered irradiation by a central point source of luminosity $L_{X}$ located at height $H_{X}$ above the disc. In their model $T_{i r r}(R) \propto\left(L_{X} H_{X}\right)^{1 / 4} R^{-3 / 4}$ for $R>>H_{X}$. The same relation was discussed in [23] for a central spherical source of radius $H_{X}$.

The radiation from the central source propagates radially outward and arrives at radius $R$ of the disc after time $\tau=R / c$. The temperature $T(R)$ increases at this radius. The peak of the resulting radiation distribution corresponds

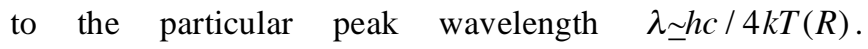
Substituting expressions for $\tau$ and $\lambda$ in Eq. (1), we obtain the time lag $\tau(\lambda)$ between the flare of the central X-ray source and the response flux variations at wavelength $\lambda$

$\tau(\lambda)=\left(\frac{3 G M \dot{M}}{8 \pi \sigma c^{3}}\right)^{1 / 3}\left[\left(\frac{h c}{4 k \lambda}\right)^{4}-\frac{1-A}{\sigma} F(\lambda)\right]^{-1 / 3}$.

Assuming that the incident X-ray flux is absorbed by the disc producing flux changes of amplitude $F(\lambda)$ at wavelengths $\lambda$ and given that the mass of black hole and accretion rate are measured, one can estimate the lag. This time lag is also related to the distance to AGN and can give the estimate of the Hubble constatnt [24]. Thus, Collier [24] considered a general temperature profile of the disc $T=T_{0}\left(R / R_{0}\right)^{-3 / 4}$ (where $T_{0}$ is the temperature at radius $R_{0}$ ). Assuming this temperature profile we can compute the continuum flux at frequency $v$ and corresponding transfer function for reprocessing of the X-ray emission into the optical range. The continuum flux can be calculated as follows (see [24, 25])

$f_{v}(\lambda)=\int_{R} B_{v} d \Omega=\frac{4 \pi R_{0}^{2} \cos i}{D^{2}}(h c)^{-5 / 3} \lambda^{-1 / 3}\left(k T_{0}\right)^{8 / 3} \frac{I_{2}(3 / 4)}{3 / 4}$,

where $B_{v}$ is the Plank function; $D$ is the luminosity distance; $i$ is the inclination angle of the disc; $I_{\mathrm{n}}(\alpha)$ is a numerical function (for the standard accretion disc $\left.I_{2}(3 / 4)=1.93\right)$. The disc transfer function depends on the time lag $\tau$ between variations of the X-ray source at height $H_{X}$ above the disc and response of the accretion disc to these variations at point with polar coordinates $(R, \theta)$. For $R>H_{X}$, the transfer function can be defined as follows (see [22])

$\psi_{v}(\tau, \lambda)=\int_{R} \frac{\partial B_{v}}{\partial T} \frac{\partial T}{\partial L_{\mathrm{X}}} \times \delta\left(\tau-\frac{R}{c}(1+\sin i \cos \theta)\right) d \Omega$,

where $\delta$ is the delta function that specifies the range of time lags for which a ring of the accretion disc of radius $R$ will be illuminated by the instantaneous flare of the X-ray source. The centroid time lag of the transfer function $<\tau>=\int \tau \psi_{v}(\tau, \lambda) d \tau / \int \psi_{v}(\tau, \lambda) d \tau$ is equivalent to the centroid of the cross-correlation function used to measure the size of the reprocessing region.

As can be seen from Eq. (3) the model of the accretion disc with the temperature profile $T \propto R^{-3 / 4}$ predicts that $f_{v} \propto \lambda^{-1 / 3}$. This is much bluer than is typically observed in AGN spectra and can be a result of contamination from the stars and hot dust in the torus. The disc spectrum can be separated from contaminants by taking a difference spectra between the brightest and faintest states of the disc: $\Delta f_{v}=f_{v}^{\max }-f_{v}^{\min }$ [24]. The observed difference spectra has been shown to exhibit a $\Delta f_{v} \propto \lambda^{-1 / 3}$ dependence.

The model spectra of the faint and bright states are defined by Eq. (3), where the temperature of the disc at radius $R_{0}$ is $T_{0}^{\min }$ and $T_{0}^{\max }$ during the faintest and the 
brightest states, respectively. Correspondingly, Eq. (4) can be rewritten with $\frac{\partial B_{v}}{\partial T} \frac{\partial T}{\partial L_{\mathrm{X}}}=B_{v}\left(T_{2}\right)-B_{v}\left(T_{1}\right) \quad$ and $T_{2}=T_{0}^{\max }\left(R / R_{0}\right)^{-3 / 4}, T_{1}=T_{0}^{\min }\left(R / R_{0}\right)^{-3 / 4}$. Thus, for the difference spectrum, the relationship between the time lag $\tau$ and continuum fluxes $f_{v}^{\max }$ and $f_{v}^{\min }$, that can be measured from observations, is expressed as

$\left(\frac{<\tau(\lambda)>}{d}\right)^{2}=\frac{f_{v}^{\max }(\lambda)}{40.1 \mathrm{~J} y}\left(\frac{\lambda}{10^{4} \AA}\right)^{3}\left(\frac{D}{\mathrm{M} p c}\right)^{2} \cos i^{-1}\left(\frac{1-\varepsilon^{3 / 2}}{1-\varepsilon}\right)^{2}$,

where $\varepsilon=f_{v}^{\min } / f_{v}^{\max }$

Hence, based on quasar light curves observed at restframe wavelengths $\lambda_{1}$ and $\lambda_{2}$, and estimates of the black hole mass and accretion rate, one can calculate a time delay, $\Delta \tau=\tau\left(\lambda_{2}\right)-\tau\left(\lambda_{1}\right)$ (using Eq. (2) and (5)), between the $\lambda_{1}$ and $\lambda_{2}$-band brightness variations, that can be expected from the model given by Eq. (1).

\section{CROSS-CORRELATION METHOD}

In order to measure interband delays we perform a crosscorrelation analysis of the multiband quasar light curves. The cross-correlation function is calculated using a modification of the CCF method (cross-correlation function method) proposed in [26, 27]. The CCF method is based on a linear interpolation in one of time series. In the method, each observed point in the time series $A\left(t_{\mathrm{i}}\right)$ is correlated with an interpolated point shifted by time lag $t_{\mathrm{i}}+\tau$ in the time series $B\left(t_{\mathrm{i}}\right)$ (where $A\left(t_{\mathrm{i}}\right)$ and $B\left(t_{\mathrm{i}}\right)$ are the quasar magnitudes at time $\left.t_{\mathrm{i}}\right)$. Thus, if there are $N$ observations, the crosscorrelation function is calculated at each lag $\tau$ for $N$ pairs of points. The ends of the both time series are extended by a constant level equal to the last points. In order to decrease interpolation errors we, following Oknyanskij [28], use a special restriction on the interpolated data: only those interpolated points that are separated from the nearest observed points by no more than some fixed value $\Delta t_{\max }$ are used for calculation of the cross-correlation coefficients. The corresponding cross-correlation function is calculated as follows:

$$
\operatorname{MCCF}(\tau)=\frac{1}{M} \sum_{\mathrm{i}, j} \frac{\left(A_{\mathrm{i}}-\bar{A}\right)\left(B_{\mathrm{j}}-\bar{B}\right)}{\sigma_{\mathrm{A}} \sigma_{\mathrm{B}}},
$$

where $M$ is the number of data pairs $\left(A_{\mathrm{i}}, B_{\mathrm{j}}\right)$ contributing to the calculation of the cross-correlation function; $\sigma_{\mathrm{A}}, \sigma_{\mathrm{B}}$ and $\bar{A}, \bar{B}$ are the standard deviations and the means of time series $A\left(t_{\mathrm{i}}\right)$ and $B\left(t_{\mathrm{i}}\right)$, respectively. In the method, only the data pairs for which $\tau-\Delta t_{\max } \leq \Delta t_{\mathrm{i} j}<\tau+\Delta t_{\max }$ (where $\Delta t_{\mathrm{i} j}=\left|t_{\mathrm{j}}-t_{\mathrm{i}}\right|$ is the time shift between the $t_{\mathrm{i}}$ point of the $A$ time series and the $t_{\mathrm{j}}$ point of the $B$ time series) are used for calculation of the MCCF function. Thus, the number of data pairs $M$ is different for each time lag $\tau$ and depends on the value of $\Delta t_{\max }$. The value of $\Delta t_{\max }$ is chosen so as to provide a compromise between the desire to decrease the interpolation errors and to find a sufficient number of data pairs to reliably calculate the cross-correlation function at a given lag.

In our calculations, the time lag values range from -100 to 100 days with a step of 0.1 days. The interpolation interval $\Delta t_{\max }$ is chosen equal to 10 days and is compared with the average sampling of the light curves during a season of observations. In order to calculate the uncertainties in time delay estimates we use the Flux Randomisation and Random Subset Selection method described in [29]. The method tests two principal sources of error: flux uncertainties and uncertainties associated with the influence of the individual data points. To test the influence of the flux uncertainties, we take the real measurement $A\left(t_{\mathrm{i}}\right)$, and then modify the flux by random Gaussian deviates based on the measured error $\sigma_{\mathrm{A}_{i}}$ (flux randomisation). The individual points in the light curves may be the result of spurious flux measurements and can considerably affect the cross-correlation analysis. Their influence is tested by a random removal of data points from the original data (random subset selection).

\section{TIME DELAY MEASUREMENTS}

\subsection{Analysis of the Multicolour Light Curves of Lensed Quasars}

In this section we analyse the published multicolor light curves of the lensed quasars Q2237+0305, SBS1520+530, HE2149-2745, HE1104-1805 and UM673 (see also [30]).

Q2237+0305. The quadruple lensed quasar Q2237+0305 discovered by Huchra et al. [31] has been extensively monitored by many groups (see [32-38]). Regular observations of Q2237+0305 started soon after the detection of the first microlensing event [39]. Since the first detection, it has been confirmed by many observations that the brightness variations seen in the light curves of the four images of the lensed quasar are mainly due to microlensing by stars in the lensing galaxy. Due to very short lensing time delays (of the order of a day) (see for example, [40-42]), the quasar's intrinsic variations should show up almost simultaneously in all images. However, similar and simultaneous brightness variations in the quasar images are a rather rare phenomenon for this system. From the historical light curves, we find only two time intervals with a similar behaviour of the light curves of Q2237+0305.

The first evidence of the intrinsic variability was reported in [32]. During the 1994 observational season, an increase in brightness of about $0.6 \mathrm{mmag} \mathrm{d} a y^{-1}$ was detected in all four images of Q2237+0305. In 2003, the quasar's intrinsic variations were observed by the OGLE and Maidanak collaborations in the $\mathrm{V}$ and $\mathrm{R}$ bands, respectively. The corresponding light curves were used to investigate the possibility of measuring the lensing time delays between the images of Q2237+0305 [19, 43]. The analysis of the interband correlations, based on the $\mathrm{V}$ and R-band light curves of images $\mathrm{A}$ and $\mathrm{C}$, showed the evidence of a time delay (9 and 16 days, respectively) between variations in 
these two bands with variations in the $\mathrm{V}$ band leading the variations in the $\mathrm{R}$ band [19].

In the present study, we analyse the OGLE V-band and the Maidanak R-band light curves [43]. As the light curves for the fainter $B$ and $D$ images of the system are characterized by larger photometric uncertainties, we consider the light curves of the brightest $\mathrm{A}$ and $\mathrm{C}$ images, $m_{V} \simeq 17.1$ and $18.1 \mathrm{mag}$, respectively. We search for a time delay between brightness variations in the $\mathrm{V}$ and $\mathrm{R}$ bands using the method of cross-correlation analysis described in Section 3. The resulting cross-correlation functions, calculated between the $\mathrm{V}$ and R-band light curves of images $\mathrm{A}$ and $\mathrm{C}$, are shown by the thick black and thin black lines in Fig. (1), respectively. The peak of the cross-correlation function, calculated between the light curves of image A, occurs at the time delay of $\tau_{\mathrm{A}}^{\text {peak }}=5.1_{-2.9}^{+0.4}$ days with a correlation coefficient of 0.98 . The peak of the crosscorrelation function between the light curves of image $\mathrm{C}$ corresponds to the time delay of $\tau_{\mathrm{B}}^{\text {peak }}=5.2_{-11.5}^{+2.9}$ days with a correlation coefficient of 0.95 . The local centroids of the cross-correlation functions calculated for the points near the main peak are $\tau_{\mathrm{A}}^{\text {cent }}=5.6_{-0.9}^{+0.7}$ and $\tau_{\mathrm{C}}^{\text {cent }}=5.1_{-4.5}^{+0.7}$ days, respectively. Here and further on in the paper, the errors quoted correspond to the 95 per cent confidence intervals.

SBS1520+530. The double lensed system SBS1520+530 [44] was monitored at the Nordic Optical Telescope (NOT) in the R band from 1999 to 2001 [45], and at Maidanak Observatory in the V and R bands between April 2003 and August 2004 [46]. The light curves of the quasar images obtained during these monitoring campaigns showed the presence of brightness variations intrinsic to the quasar. These light curves were used to estimate the lensing time delay in images of SBS1520+530 (see [45, 46]).

In order to explore the interband correlations, we analyse the Maidanak $\mathrm{V}$ and R-band light curves of images $\mathrm{A}$ $\left(m_{V} \simeq 18.5 \mathrm{mag}\right)$ and $\mathrm{B}\left(m_{V} \simeq 19.4 \mathrm{mag}\right)$ of SBS1520+530 presented in [46]. The lensed system SBS1520+530 is also found to exhibit slow long-term microlensing variations superimposed on the quasar variations [46, 47]. The microlensing variations, which are also wavelengthdependent, can be an additional source of noise which might lead to a bias in interband time delay estimates. The analysis of the difference between the light curves of the quasar images after correction for lensing time delay and magnitude offset showed, that within statistical uncertainty, the two quasar light curves were identical during the Maidanak observations [46]. This indicates that the microlensing component of brightness variations in the analysed light curves is small. Thus, we do not expect that microlensing will seriously bias the time delay estimate.

The resulting cross-correlation functions are shown in Fig. (1). The cross-correlation function between the $\mathrm{R}$ and V-band light curves of image A (shown by the thick black

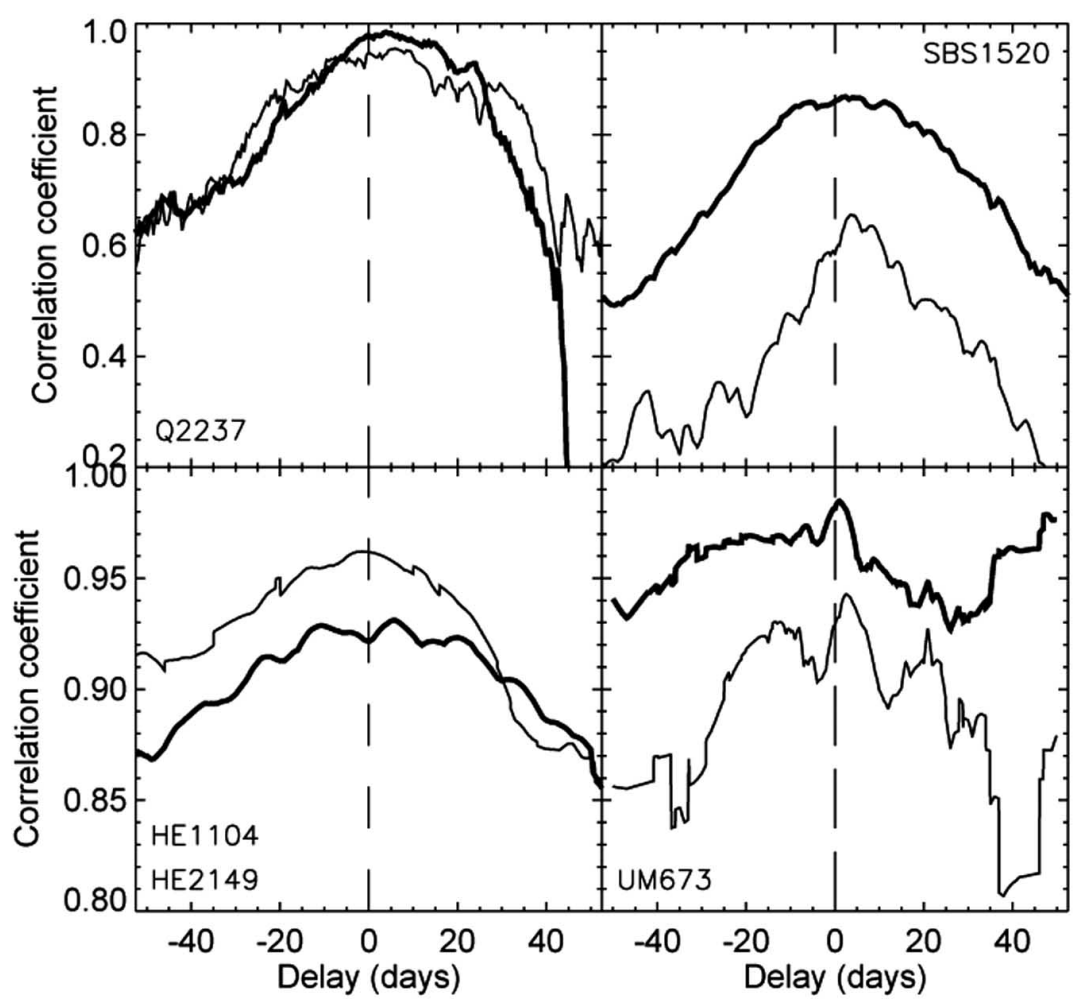

Fig. (1). Upper left panel: cross-correlation functions calculated between the $\mathrm{V}$ and R-band light curves of images $\mathrm{A}$ (thick black line) and C (thin black line) of the lensed quasar Q2237+0305. Upper right panel: cross-correlation functions calculated between the V and R-band light curves of images A (thick black line) and B (thin black line) of the lensed quasar SBS1520+530. Bottom left panel: cross-correlation functions between the V and i-band light curves of image A of the lensed quasar HE2149-2745 (thick black line) and between the B and R-band light curves of image A of the lensed quasar HE1104-1805 (thin black line). Bottom right panel: cross-correlation functions between the V and I-band light curves of images A (thick black line) and B (thin black line) of the lensed quasar UM673. The vertical dashed lines correspond to zero time delay. 
line), has a very broad peak with a maximum at the time delay $\tau_{\mathrm{A}}^{\text {peak }}=4.4_{-9.5}^{+2.4}$ days and a correlation coefficient of 0.87 . The peak of the cross-correlation function, calculated between the light curves of image B (shown by the thin black line), occurs at the time delay of $\tau_{\mathrm{B}}^{\text {peak }}=6.1_{-6.7}^{+2.2}$ days with a correlation coefficient of 0.66 . The local centroids of the cross-correlation functions are $\tau_{\mathrm{A}}^{\text {cent }}=2.3_{-2.5}^{+2.5}$ and $\tau_{\mathrm{B}}^{\text {cent }}=2.8_{-2.3}^{+3.7}$ days for the image $\mathrm{A}$ and $\mathrm{B}$ light curves, respectively.

HE2149-2745. The double lensed quasar HE2149-2745 [48] was monitored with the 1.5-m Danish Telescope (ESOLa Silla) in the V and Gunn i bands between October 1998 and December 2000. During the observations, the quasar showed slow long-term intrinsic brightness variations that gave a lensing time delay of $103 \pm 12$ days [49]. External microlensing variations were not reliably detected during time interval covered by the observations $[47,49]$.

In this study, we analyse the $\mathrm{V}$ and i-band light curves of image A of HE2149-2745 ( $m_{V} \simeq 17 \mathrm{mag}$ ). The B image light curves are noisier and can not be used for our analysis. The resulting cross-correlation function, shown by the thick black line in Fig. (1), has a very broad peak with several local maxima. The maximum correlation coefficient of 0.93 corresponds to a time delay of $\tau_{\mathrm{A}}^{\text {peak }}=4.0_{-13.0}^{+10.0}$ days. The centroid time delay is $\tau_{\mathrm{A}}^{\text {cent }}=2.5_{-6.3}^{+4.8}$ days.

HE1104-1805. The double lensed quasar HE1104-1805 [50] was extensively monitored both spectroscopically and optically with the aim of measuring lensing time delay and studying microlensing in the system [51-55]. Poindexter et al. [55] presented the results of multicolour observations of the quasar monitored with the 1.3-m SMARTS telescope between December 2003 and May 2006. These data were used to improve the time delay between quasar images, previously measured in $[53,54]$. The difference light curves showed the presence of significant microlensing variations in one of the quasar images on the timescale of the lensing time delay ( $\sim 150$ days). These microlensing variations may lead to additional uncertainties in the interband time delay analysis.

We analyse the better-sampled B and R-band light curves of image A of HE1104-1805 ( $m_{R} \simeq 16.5 \mathrm{mag}$ ). The resulting cross-correlation function is shown by the thin black line in Fig. (1). We find that the peak of the cross-correlation function occurs at the time delay of $\tau_{\mathrm{A}}^{\text {peak }}=-3.1_{-8.7}^{+5.4}$ days with a correlation coefficient of 0.97 . The corresponding centroid time delay is $\tau_{\mathrm{A}}^{\mathrm{cent}}=-4.3_{-3.4}^{+3.1}$ days. In our analysis, the sign of the delay depends on which of the two light curves is chosen for interpolation. Usually, it is the better-sampled light curve. The observed points from the real light curve are then correlated with the points from the interpolated light curve, shifted by a time lag. Thus, the sign of the time delay between the interpolated R-band and observed B-band light curves is negative and vice versa, between the interpolated B-band and observed R-band light curves it is positive. The sign "-" here means that the brightness variations in the R- band light curve, chosen for interpolation, follow the brightness variations in the B-band light curve.

UM673 (Q0142-100). The double lensed quasar UM673 [56] was monitored with the $1.5-\mathrm{m}$ telescope at Maidanak Observatory in the V, R and I bands between August 2003 and November 2005. During the observations, the quasar showed intrinsic brightness variations seen in both images of UM673 [57].

Here, we analyse the $\mathrm{V}$ and I-band light curves of images $\mathrm{A}$ and $\mathrm{B}, \quad m_{V} \simeq 16.2$ and $18.3 \mathrm{mag}$, respectively. The resulting cross-correlation functions are shown in Fig. (1). The peak of the cross-correlation function, calculated between the $\mathrm{V}$ and I-band light curves of image A (shown by the thick black line), occurs at the time delay of $\tau_{\mathrm{A}}^{\text {peak }}=1.2_{-11.4}^{+2.3} \quad$ days. The corresponding correlation coefficient is 0.98 . The peak of the cross-correlation function between the light curves of image $B$ (shown by the thin black line) occurs at the time delay of $\tau_{\mathrm{B}}^{\text {peak }}=2.9_{-8.1}^{+3.9}$ days with a correlation coefficient of 0.94 . The local centroids of the cross-correlation functions are $\tau_{\mathrm{A}}^{\text {cent }}=1.0_{-4.4}^{+5.8}$ and $\tau_{\mathrm{B}}^{\text {cent }}=2.1_{-3.5}^{+1.8}$ days, respectively.

In Table 1 we summarize our measurements as well as the previously measured interband time delay in Q0957+561 [17]. In all cases, we find that the brightness variations at longer wavelengths may follow the brightness variations at shorter wavelengths by a few days. In Table 1 we also present the time delays $\tau^{\text {model }}$ that might be expected from a model of a standard accretion disc irradiated by the central variable source of X-ray radiation (see Eq. 2 and 5). The predicted delays are estimated for the light curves of the A images of the lensed quasars. Since the interband time delays are connected with the physics of the quasar, these delays are expected to be the same in other images. The possible differences might be due to the microlensing variations superimposed on the quasar's intrinsic variations, and also due to extinction by the dust in the lensing galaxy (see [58]). In the case of double lensed quasars, image A, which is usually further away from the lensing galaxy, is likely to be less affected by microlensing and dust extinction.

The variability characteristics of the light curves of the A images (amplitudes, maximum and minimum fluxes) are listed in Table 2. In order to determine the quasar's intrinsic fluxes we divide the observed fluxes by an image magnification factor. The magnifications of the images in the lensed systems Q2237+0305 and HE11041805 were calculated using parameters of the macrolens models, local convergence $\kappa$ and shear $\gamma$, published in [41, 52]. In order to estimate the magnification of image A for the lensed systems SBS1520+530, HE2149-2745 and UM673, we adopt the likely values for local parameters $(\kappa=0.46$, and $\gamma=0.54)$ (see [59]). We note, that the actual magnification of an image is a sum of macrolens magnification and magnification due to microlensing by the stars of the lensing galaxy. For the most of the lensed quasars in our sample, we can assume that there are absent or very small microlensing variations in image A. However, magnification due to microlensing might be 
Table 1. Interband Time Delays (in the Observer Rest Frame) Obtained from the Cross-Correlation Analysis of the Lensed Quasar Light Curves Compared with the Delays $\tau^{\text {model }}$ Expected from Reprocessing in the Standard Accretion Disc. The $\tau^{\text {model }}$ Time Delays were Calculated Using Eq. 2 and 5

\begin{tabular}{|c|c|c|c|c|c|c|c|}
\hline Object & $\mathrm{z}$ & Bands & $\begin{array}{c}\tau^{\text {peak }} \\
\text { (days) }\end{array}$ & $\begin{array}{c}\tau^{\text {cent }} \\
\text { (days) }\end{array}$ & $\begin{array}{c}\tau^{\text {model }} \text { (Eq. 5) } \\
\text { (days) }\end{array}$ & $M_{9} \dot{M}$ & $\begin{array}{c}\tau^{\text {model }} \text { (Eq. 2) } \\
\text { (days) }\end{array}$ \\
\hline Q0957+561 [17] & 1.41 & $g r$ & & & & & \\
\hline $\mathrm{AB}$ & & & $4.1_{-4.1}^{+0.9}$ & $3.4_{-1.4}^{+1.5}$ & 3.4 & 0.82 & 8.8 \\
\hline $\mathrm{Q} 2237+0305$ & 1.69 & $V R$ & & & & & \\
\hline A & & & $5.1_{-2.9}^{+0.4}$ & $5.6_{-0.9}^{+0.7}$ & 1.1 & $0.04 \div 0.25$ & $1.4 \div 4.7$ \\
\hline $\mathrm{C}$ & & & $5.2_{-11.5}^{+2.9}$ & $5.1_{-4.5}^{+0.7}$ & 2.3 & & \\
\hline SBS $1520+530$ & 1.86 & $V R$ & & & & & \\
\hline A & & & $4.4_{-9.5}^{+2.4}$ & $2.3_{-2.5}^{+2.5}$ & 0.8 & 0.04 & 1.4 \\
\hline B & & & $6.1_{-6.7}^{+2.2}$ & $2.8_{-2.3}^{+3.7}$ & & & \\
\hline HE2149-2745 & 2.03 & $V i$ & & & & & \\
\hline A & & & $4.0_{-13.0}^{+10.0}$ & $2.5_{-6.3}^{+4.8}$ & 1.0 & 0.49 & 12.8 \\
\hline HE1104-1805 & 2.32 & $B R$ & & & & & \\
\hline A & & & $3.1_{-8.7}^{+5.4}$ & $4.3_{-3.4}^{+3.1}$ & 3.3 & 0.07 & 3.8 \\
\hline UM673 & 2.72 & $V I$ & & & & & \\
\hline A & & & $1.2_{-11.4}^{+2.3}$ & $0.8_{-4.4}^{+5.8}$ & 10.7 & 0.71 & 9.5 \\
\hline B & & & $2.9_{-8.1}^{+3.9}$ & $3.1_{-3.5}^{+1.8}$ & & & \\
\hline
\end{tabular}

significant in the images of Q2237+0305. The time delays (see Eq. 5) calculated based on the properties of images A and $\mathrm{C}$ (fluxes and macrolens magnifications) differ by about one day. This is probably related to the presence of additional magnification caused by stars close to the line of sight of the images. In the model we also adopt the disc inclination angle $i=45^{\circ}$. The luminosity distances are computed assuming the Hubble constant $H_{0}=75 \mathrm{~km} \mathrm{~s}^{-1} \mathrm{Mpc}^{-1}$, deceleration parameter $q 0=0.5$ and cosmological constant equal to zero. In the last two columns of Table $\mathbf{1}$ we present the product of the black hole mass $M_{9}$ in $10^{9} M_{\odot}$ and accretion rate $\dot{M}$ as a fraction of the Eddington accretion rate, and time delays $\tau^{\text {model }}$ calculated from Eq. 2. For the lensed quasars SBS1520+530,

Table 2. Amplitudes of Variations, and Maximum and Minimum fluxes (mJy) Estimated From the Image A Quasar Light Curves

\begin{tabular}{lcccc}
\hline Object & Bands & $\begin{array}{c}\Delta m \\
(\mathrm{mag})\end{array}$ & $\begin{array}{c}f_{\max } \\
(\mathrm{mJy})\end{array}$ & $\begin{array}{c}f_{\min } \\
(\mathrm{mJy})\end{array}$ \\
\hline Q2237+0305 & $\mathrm{V}$ & 0.18 & 0.39 & 0.33 \\
& $\mathrm{R}$ & 0.12 & 0.29 & 0.25 \\
SBS1520+530 & $\mathrm{V}$ & 0.08 & 0.13 & 0.12 \\
& $\mathrm{R}$ & 0.06 & 0.10 & 0.09 \\
HE2149-2745 & $\mathrm{V}$ & 0.15 & 0.13 & 0.12 \\
& $\mathrm{i}$ & 0.12 & 0.07 & 0.06 \\
HE1104-1805 & $\mathrm{B}$ & 0.27 & 0.42 & 0.33 \\
& $\mathrm{R}$ & 0.29 & 0.24 & 0.18 \\
$\mathrm{UM} 673$ & $\mathrm{~V}$ & 0.20 & 1.19 & 0.99 \\
& $\mathrm{I}$ & 0.15 & 0.93 & 0.81 \\
\hline
\end{tabular}

HE2149-2745, HE1104-1805 and UM673 we use the lower limits (calculated with correction for lensing magnifications) of black hole masses and accretion rates published in [60]. In order to calculate $\tau^{\text {model }}$ ' for the lensed quasar Q2237+0305 we adopt the black hole mass estimate given in [61] and $M_{\simeq}$ 0.1 .

\subsection{Analysis of the Multicolour Light Curves of Non- lensed Quasars}

In order to complete our analysis of the interband time delays in the lensed quasars, we also analyse the light curves of nonlensed quasars from the MACHO database [62]. The MACHO Project monitored the Magellanic Clouds with the aim of detecting microlensing events (see [63]). The collected photometric data include the 7.5-year V and R-band light curves of 12 known quasars and AGN as well as the light curves of 47 new quasars discovered during the project. The quasars cover the redshift interval $0.2<z<2.8$. The average sampling of the light curves of 2-10 days is good enough to detect delays on the order of a day. For the time delay analysis we select the better-sampled light curves of 10 quasars in the redshift interval $1<z<2.3$ so that, the analysed rest-frame wavelengths are nearly the same for the lensed and nonlensed quasars. We also assume that the luminosity range of the MACHO quasars covers the luminosity range of the lensed quasars. The MACHO quasars and their redshifts and absolute magnitudes $M_{V}$ are presented in Table 3. The magnitude measurements with photometric errors of more than 5 per cent are excluded from analysis. The results of the cross-correlation analysis are presented in Fig. (2), and in the fourth and fifth columns of Table 3. The correlation coefficients corresponding to the peak of the cross- 


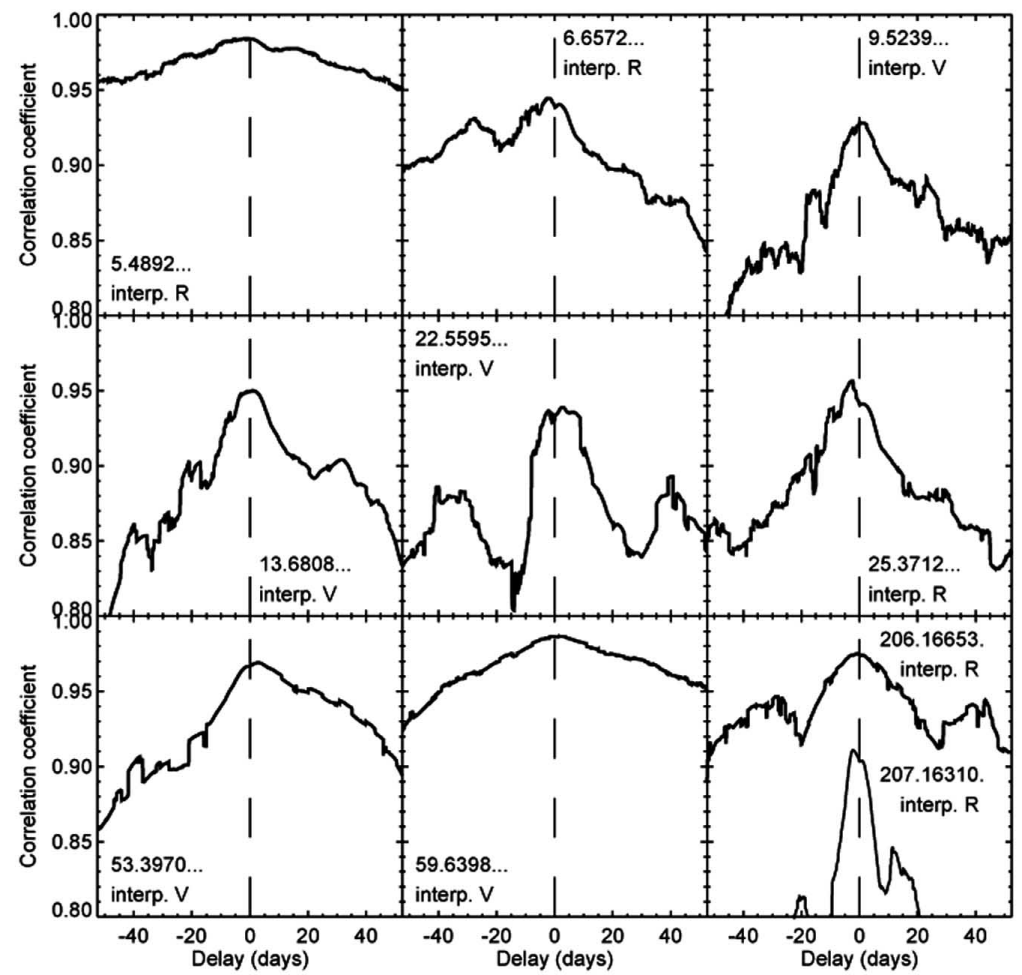

Fig. (2). Cross-correlation functions between the V and R-band quasar light curves. The sign of the delay corresponding to the peak of the cross-correlation function depends on which of the two light curves is chosen for interpolation. In all cases, the peak time delay is consistent with the R-band variations following the V-band variations. The vertical dashed lines correspond to zero time delay.

Table 3. MACHO Quasars. Results of Cross-Correlation Analysis Compared with Predictions from Reprocessing in the Standard Accretion Disc

\begin{tabular}{lcccccc}
\hline MACHO ID & $\mathrm{z}$ & $M_{\mathrm{V}}$ & $\begin{array}{c}\tau^{\text {peak }} \\
\text { (days) }\end{array}$ & $\begin{array}{c}\tau^{\text {cent }} \\
\text { (days) }\end{array}$ & $r^{\text {max }}$ & $\begin{array}{c}\tau^{\text {model}}(\text { Eq. 5) } \\
\text { (days) }\end{array}$ \\
\hline 5.4892 .1971 & 1.58 & -26.73 & $2.3_{-0.8}^{+6.6}$ & $2.4_{-4.2}^{+2.0}$ & 0.984 & 2.4 \\
6.6572 .268 & 1.81 & -27.22 & $2.2_{-9.4}^{+3.0}$ & $2.5_{-1.4}^{+2.6}$ & 0.944 & 2.3 \\
9.5239 .505 & 1.30 & -25.48 & $1.6_{-5.1}^{+4.4}$ & $1.7_{-7.0}^{+4.2}$ & 0.947 & 1.5 \\
13.6808 .521 & 1.64 & -26.26 & $1.9_{-7.6}^{+2.4}$ & $2.0_{-5.3}^{+6.1}$ & 0.950 & 1.9 \\
22.5595 .1333 & 1.15 & -25.77 & $0.8_{-3.4}^{+7.6}$ & $1.5_{-6.8}^{+2.8}$ & 0.945 & 1.3 \\
25.3712 .72 & 2.17 & -27.20 & $2.7_{-3.2}^{+2.4}$ & $2.8_{-1.8}^{+3.2}$ & 0.956 & 3.2 \\
53.3970 .140 & 2.04 & -27.14 & $3.5_{-6.0}^{+4.0}$ & $2.6_{-3.4}^{+6.2}$ & 0.969 & 2.8 \\
59.6398 .185 & 1.64 & -25.95 & $2.4_{-12.1}^{+2.2}$ & $1.5_{-3.8}^{+6.8}$ & 0.983 & 1.8 \\
206.16653 .987 & 1.05 & -24.57 & $1.3_{-2.6}^{+8.8}$ & $1.3_{-2.2}^{+6.0}$ & 0.973 & 0.7 \\
207.16310 .1050 & 1.47 & -25.80 & $2.2_{-0.6}^{+7.2}$ & $2.3_{-1.0}^{+5.6}$ & 0.971 & 1.5 \\
\hline
\end{tabular}

correlation function, $r^{\max }$, are given in the sixth column of Table 3. The time delays $\tau$ moder expected from the reprocessing model (the last column of Table 3 ) are calculated using the observed minimum and maximum fluxes estimated from the $\mathrm{V}$ and R-band quasar light curves (see Table 4).

\section{SUMMARY}

In this study, we search for possible interband time delays in a sample of lensed quasars optically monitored in one or more bands. From cross-correlation analysis of the quasar light curves we find that the brightness variations at longer wavelengths may follow the brightness variations at shorter wavelengths in the observer rest frame by a few days (see Table 1). An independent analysis of multiwavelength light curves of nonlensed quasars in the same redshift interval confirms the evidence of a time delay, although the 95 per cent confidence intervals are large and the detected time delays need further confirmation.

In order to compare the observed delays with the delays that might be expected from reprocessing in the accretion disc, we consider a model of the standard accretion disc irradiated by the central variable source of X-ray radiation. This 
Table 4. Amplitudes of Variations, and Maximum and Minimum fluxes (mJy) Estimated from the Image A Quasar Light Curves

\begin{tabular}{|c|c|c|c|c|}
\hline Object & Bands & $\begin{array}{c}\Delta m \\
\text { (mag) }\end{array}$ & $\begin{array}{c}f_{\max } \\
(\mathrm{mJy})\end{array}$ & $\begin{array}{c}f_{\min } \\
(\mathrm{mJy})\end{array}$ \\
\hline \multirow[t]{2}{*}{5.4892 .1971} & $\mathrm{~V}$ & 0.45 & 0.47 & 0.31 \\
\hline & $\mathrm{R}$ & 0.40 & 0.42 & 0.29 \\
\hline \multirow[t]{2}{*}{6.6572 .268} & $\mathrm{~V}$ & 0.25 & 0.54 & 0.43 \\
\hline & $\mathrm{R}$ & 0.18 & 0.43 & 0.37 \\
\hline \multirow[t]{2}{*}{9.5239 .505} & $\mathrm{~V}$ & 0.30 & 0.21 & 0.16 \\
\hline & $\mathrm{R}$ & 0.26 & 0.20 & 0.15 \\
\hline \multirow[t]{2}{*}{13.6808 .521} & $\mathrm{~V}$ & 0.34 & 0.28 & 0.20 \\
\hline & $\mathrm{R}$ & 0.25 & 0.24 & 0.19 \\
\hline \multirow[t]{2}{*}{22.5595 .1333} & $\mathrm{~V}$ & 0.46 & 0.33 & 0.22 \\
\hline & $\mathrm{R}$ & 0.42 & 0.28 & 0.19 \\
\hline \multirow[t]{2}{*}{25.3712 .72} & V & 0.25 & 0.46 & 0.37 \\
\hline & $\mathrm{R}$ & 0.20 & 0.41 & 0.34 \\
\hline \multirow[t]{2}{*}{53.3970 .140} & $\mathrm{~V}$ & 0.25 & 0.47 & 0.37 \\
\hline & $\mathrm{R}$ & 0.22 & 0.40 & 0.33 \\
\hline \multirow[t]{2}{*}{59.6398 .185} & $\mathrm{~V}$ & 0.62 & 0.23 & 0.13 \\
\hline & $\mathrm{R}$ & 0.50 & 0.20 & 0.12 \\
\hline \multirow[t]{2}{*}{206.16653 .987} & $\mathrm{~V}$ & 0.45 & 0.14 & 0.10 \\
\hline & $\mathrm{R}$ & 0.30 & 0.12 & 0.09 \\
\hline \multirow[t]{2}{*}{207.16310 .1050} & V & 0.23 & 0.22 & 0.18 \\
\hline & $\mathrm{R}$ & 0.16 & 0.19 & 0.17 \\
\hline
\end{tabular}

model accounts for both intrinsic emission from the accretion disc and reprocessing of the X-ray emission. Our comparison of the time delays with the model predictions shows that the observed time delays are rather consistent with the scenario where reprocessing of the X-rays is the primary mechanism of quasar variability, at least on the timescales investigated by us. We also find (mainly from analysis of the MACHO sample of quasars) that the interband delays are longer for the higher luminosity quasars. This result is in agreement with the lag-luminosity relationship found for nearby Seyfert galaxies [12].

In our study we explore the interband time delays in the quasars from the redshift range $1.0<z<2.7$. These delays help us to understand the mechanisms of variability. The interband time delays can also be used to study the structure of the emission region of the quasars. As the interband delays are wavelength-dependent as $\tau \propto \lambda^{\xi}$ (with $\xi=4 / 3$ for the standard accretion disc), the new multiwavelength broadband observations of quasars and AGN can also help us to place constraints on accretion disc models.

\section{ACKNOWLEDGEMENTS}

This research was supported by the Russian Foundation for Basic Research (RFBR) Grant No. 09-02-00244a, and by the Taiwan National Science Council Grant No. 96-2811-M008-033.

\section{REFERENCES}

[1] Clavel J, Nandra K, Makino F, et al. Correlated hard X-ray and ultraviolet variability in NGC 5548. Astrophys J 1992; 393: 11325 .

[2] Edelson RA, Alexander T, Crenshaw DM, et al. Multiwavelength observations of short-timescale variability in NGC 4151. IV.
Analysis of multiwavelength continuum variability. Astrophys J 1996; 470: 364-77.

[3] Krolik JH, Horne K, Kallman TR, Malkan MA, Edelson RA, Kriss GA. Ultraviolet variability of NGC 5548 -Dynamics of the continuum production region and geometry of the broad-line region. Astrophys J 1991; 371: 541-62.

[4] Arevalo P, Uttley P, Kaspi S, Breedt E, Lira P, McHardy IM. Correlated X-ray/optical variability in the quasar MR2251-178. Mon Not R Astron Soc 2008; 389: 1479-88.

[5] Arevalo P, Uttley P, Lira P, Breedt E, McHardy IM, Churazov E. Correlation and time delays of the X-ray and optical emission of the Seyfert galaxy NGC 3783. Mon Not R Astron Soc 2009; 397: 2004-14.

[6] Breedt E, Arevalo P, McHardy IM, et al. Long-term optical and Xray variability of the Seyfert galaxy Markarian 79. Mon Not R Astron Soc 2009; 394: 427-37.

[7] Bachev R, Grupe D, Boeva S, et al. Studying X-ray reprocessing and continuum variability in quasars: PG $1211+143$. Mon Not R Astron Soc 2009; 399, 750-61.

[8] Stalin CS, Gupta AC, Gopal. K, Witta PJ, Sagar R. Intranight optical variability of BL Lacs, radio-quiet quasars and radio-loud quasars. Mon Not R Astron Soc 2005; 356: 607-14.

[9] Czerny B, Siemiginowska A, Janiuk A, Gupta AC. The nature of the intranight variability of radio-quiet quasars. Mon Not R Astron Soc 2008; 386: 1557-67.

[10] Collier SJ, Horne K, Kaspi S, et al. Steps toward determination of the size and structure of the broad-line region in active galactic nuclei. XIV. Intensive optical spectrophotometric observations of NGC 7469. Astrophys J 1998; 500: 162-72.

[11] Oknyanskij VL, Horne K, Lyuty VM, Sadakane K, Honda S, Tanabe S. Time delays in NGC 4151. Astron Soc Pac Conf Ser 2003; 290: $119-20$

[12] Sergeev SG, Doroshenko VT, Golubinskiy YuV, Merkulova NI, Sergeeva EA. Lag-luminosity relationship for interband lags between variations in $\mathrm{B}, \mathrm{V}, \mathrm{R}$, and $\mathrm{I}$ bands in active galactic nuclei. Astrophys J 2005; 622: 129-35.

[13] Magdis G, Papadakis IE. An optical variability study of quasars using the MACHO light curves. Astron Soc Pac Conf Ser 2006; 360: 37-9.

[14] Bachev RS. Quasar optical variability: searching for interband time delays. Astron Astrophys 2009; 493: 907-11.

[15] Giveon U, Maoz D, Kaspi S, Netzer H, Smith PS. Long-term optical variability properties of the Palomar-Green quasars. Mon Not R Astron Soc 1999; 306: 637-54

[16] Refsdal S. On the possibility of determining Hubble's parameter and the masses of galaxies from the gravitational lens effect. Mon Not R Astron Soc 1964; 128: 307-10.

[17] Collier S. Evidence for accretion disc reprocessing in QSO 0957+561. Mon Not R Astron Soc 2001; 325: 1527-32.

[18] Shalyapin VN, Goicoechea LJ, Koptelova E, Ullan A, Gil-Merino R. New two-colour light curves of Q0957+561: time delays and the origin of intrinsic variations. Astron Astrophys 2008; 492: 401-10.

[19] Koptelova EA, Oknyanskij VL, Shimanovskaya EV. Determining time delay in the gravitationally lensed system QSO2237+0305. Astron Astrophys 2006; 452: 37-46.

[20] Shakura NI, Sunyaev RA. Black holes in bynary systems. Observational appearance. Astron Astrophys 1973; 24: 337-55.

[21] Frank J, Andrew K, Derek R. Accretion power in astrophysics. Cambridge: Cambridge University Press 2002.

[22] Berkley, AJ, Kazanas D, Ozik J. Modeling the X-ray ultraviolet correlations in NGC 7469. Astrophys J 2000; 535: 712-20.

[23] Rokaki E, Magnan C. Correlated variability of lines and continuum by disc reprocessing in NGC5548. Astron Astrophys 1992; 261: 41-6.

[24] Collier S, Horne K, Wanders I, Peterson BM. A new direct method for measuring the Hubble constant from reverberating accretion discs in active galaxies. Mon Not R Astron Soc 1999; 302: L24-8.

[25] Cackett EM, Horne K, Winkler H. Testing thermal reprocessing in active galactic nuclei accretion discs. Mon Not R Astron Soc 2007; 380: 669-2.

[26] Gaskell CM, Sparke LS. Line variations in quasars and Seyfert galaxies. Astrophys J 1986; 305: 175-6.

[27] Gaskell CM, Peterson BM. The accuracy of cross-correlation estimates of quasar emission-line region sizes. Astrophys J Suppl Ser 1987; 65: 1-11. 
[28] Oknyanskij VL. Delayed infrared emission of the nucleus of NGC 4151. Astron Lett 1993; 19: 416-9.

[29] Peterson BM, Wanders I, Horne K, et al. On uncertainties in crosscorrelation lags and the reality of wavelength-dependent continuum lags in active galactic nuclei. Publ Astron Soc Pac 1998; 110: 66070.

[30] Koptelova E, Oknyanskij V, Artamonov B, Chen WP. Multiwavelength observations of lensed quasars: searching for interband time delays Procedure: Proceedings of the Frascati Workshop Multifrequency Behaviour of High Energy Cosmic Sources; 2009 May. Societa Astronomica Italiana, Memorie, Frascati, Italy 2010; 81: pp. 138-43.

[31] Huchra J, Gorenstein M, Kent S, et al. 2237 + 0305: A new and unusual gravitational lens. Astrophys J 1985; 90: 691-696.

[32] Ostensen R, Refsdal S, Stabell R, et al. Monitoring of the Einstein Cross with the Nordic Optical Telescope. Astron Astrophys 1996; 309: 59-64.

[33] Wozniak PR, Alard C, Udalski A, et al. The Optical Gravitational Lensing Experiment Monitoring of QSO 2237+0305. Astrophys J 2000; 529: 88-92.

[34] Alcalde D, Mediavilla E, Moreau O, et al. QSO 2237+0305 VR light curves from Gravitational LensES International Time Project Optical Monitoring. Astrophys J 2002; 572: 729-734.

[35] Schmidt RW, Kundic T, Pen U-L, et al. Optical monitoring of the gravitationally lensed quasar Q2237+0305 from APO between June 1995 and January 1998. Astron Astrophys 2002; 392: 773-779.

[36] Vakulik VG, Schild RE, Dudinov VN, et al. Color effects associated with the 1999 microlensing brightness peaks in gravitationally lensed quasar Q2237+0305. Astron Astrophys 2004; 420: 447-457.

[37] Koptelova E, Shimanovskaya E, Artamonov B, et al. Image reconstruction technique and optical monitoring of the QSO2237+0305 from Maidanak Observatory in 2002-2003. Mon Not R Astron Soc 2005; 356: 323-330.

[38] Udalski A, Szymanski MK, Kubiak M, et al. The Optical Gravitational Lensing Experiment. OGLE-III long term monitoring of the gravitational lens QSO 2237+0305. Acta Astron 2006; 56: 293305.

[39] Irwin MJ, Webster RL, Hewett PC, Corrigan RT, Jedrzejewski RI. Photometric variations in the Q2237 + 0305 system -First detection of a microlensing event. Astrophys J 1989; 98: 1989-94.

[40] Wambsganss J, Paczynski B. Parameter degeneracy in models of the quadruple lens system Q2237+0305. Astron J 1994; 108: 115662.

[41] Schmidt R, Webster RL, Lewis GF. Weighing a galaxy bar in the lens Q2237 + 0305. Mon Not R Astron Soc 1998; 295: 488-96.

[42] Dai X, Chartas G, Agol E, Bautz MW, Garmire GP. Chandra Observations of QSO 2237+0305. Astrophys J 2003; 589: 100-110.

[43] Vakulik V, Schild R, Dudinov V et al. Observational determination of the time delays in gravitational lens system Q2237+0305. Astron Astrophys 2006; 447: 905-13.

[44] Chavushyan VH, Vlasyuk VV, Stepanian JA, Erastova LK. SBS 1520+530: a new gravitationally lensed system at $z=1.855$. Astron Astrophys 1997; 318: L67-70.

[45] Burud I, Hjorth J, Courbin F, et al. Time delay and lens redshift for the doubly imaged BAL quasar SBS 1520+530. Astron Astrophys 2002; 391: 481-6.
[46] Gaynullina ER, Schmidt RW, Akhunov T, et al. Microlensing in the double quasar SBS 1520+530. Astron Astrophys 2005; 440: 538.

[47] Paraficz D, Hjorth J, Burud I, Jakobsson P, Eliasdottir A. Microlensing variability in time-delay quasars. Astron Astrophys 2006; 455: L1-4.

[48] Wisotzki L, Kohler T, Lopez S, Reimers D. Discovery of a new gravitationally lensed QSO with broad absorption lines. Astron Astrophys 1996; 315: 405-8.

[49] Burud I, Courbin F, Magain P, et al. An optical time-delay for the lensed BAL quasar HE 2149-2745. Astron Astrophys 2002; 383 : 71-81.

[50] Wisotzki L, Koehler T, Kayser R, Reimers D. The new double QSO HE 1104-1805: Gravitational lens with microlensing or binary quasar? Astron Astrophys 1993; 278: L15-8.

[51] Wisotzki L, Wucknitz O, Lopez S, Sorensen AN. First estimate of the time delay in HE 1104-1805. Astron Astrophys 1998; 339: L73-6.

[52] Schechter PL, Udalski A, Szymanski M, et al. Microlensing of relativistic knots in the quasar HE $11041805 \mathrm{AB}$. Astrophys J 2003; 584: 657-3.

[53] Ofek EO, Maoz D. Time-delay measurement of the lensed quasar HE 1104-1805. Astrophys J 2003; 594: 101-6.

[54] Wyrzykowski L, Udalski A, Schechter PL, et al. The Optical Gravitational Lensing Experiment. Optical monitoring of the gravitationally lensed quasar HE1104-1805 in 1997-2002. Acta Astron 2003; 53: 229-40.

[55] Poindexter S, Morgan N, Kochanek CS, Falco EE. Mid-IR observations and a revised time delay for the gravitational lens system quasar HE 1104-1805. Astrophys J 2007; 660: 146-51.

[56] MacAlpine GM, Feldman FR. Discovery and spectrophotometry of high-redshift quasars. Astrophys J 1982; 261: 412-21.

[57] Koptelova E, Oknyanskij VL, Artamonov BP, Burkhonov O. Intrinsic quasar variability and time delay determination in the lensed quasar UM673. Mon Not R Astron Soc 2010; 401: 2805-15.

[58] Falco EE, Impey CD, Kochanek CS, et al. Dust and extinction curves in galaxies with $\mathrm{z}>0$ : The interstellar medium of gravitational lens galaxies. Astrophys J 1999; 523: 617-32.

[59] Wyithe JSB, Turner EL. Cosmological microlensing statistics: variability rates for quasars and gamma-ray burst afterglows and implications for macrolensing magnification bias and flux ratios. Astrophys J 2002; 575: 650-66.

[60] Peng CY, Impey CD, Rix H-W, et al. Probing the coevolution of supermassive black holes and galaxies using gravitationally lensed quasar hosts. Astrophys J 2006; 649: 616-34.

[61] Kochanek CS. Quantitative interpretation of quasar microlensing light curves. Astrophys J 2004; 605: 58-77.

[62] Geha M, Alcock C, Allsman RA, et al. Variability-selected quasars in MACHO project Magellanic Cloud fields. Astron J 2003; 125: 112.

[63] Alcock C, Allsman RA, Alves D, et al. The MACHO project Large Magellanic Cloud microlensing results from the first two years and the nature of the Galactic dark halo. Astrophys J 1997; 486: 697726.

(C) Koptelova and Oknyanskij; Licensee Bentham Open.

This is an open access article licensed under the terms of the Creative Commons Attribution Non-Commercial License (http://creativecommons.org/licenses/by-nc/3.0/) which permits unrestricted, non-commercial use, distribution and reproduction in any medium, provided the work is properly cited. 